differences in obesity levels between YP living in the poorest areas of the country and the richest.

- YP in the UK have a higher burden of disease from long term conditions such as diabetes than some of their peers in other countries.

- The UK is in the middle of the pack compared to other countries on some indicators for YP, including cancer mortality, smoking, alcohol consumption and cannabis use. Trends in health-related behaviours such as smoking, and alcohol consumption have been falling internationally in recent years, reflected in the UK statistics.

- The UK has some of the lowest rates of road traffic injury deaths, which have also been steadily improving over time.

- Overall, the UK performs in the top third of countries on mortality rates for 10-to 19-year-olds. Recently, however, progress has stalled and for YP aged 20-24 got worse between 2013 and 2016.

Conclusions Although there are some positive findings, the UK's performance on many indicators for this age-group are lagging behind that of similar high-income countries. An increased policy focus is required on this age-group if these worrying trends are to be reversed.

\section{POLYCYSTIC OVARIAN SYNDROME IN ADOLESCENTS: DISCOVERY PROTEOMICS AND THE SEARCH FOR NOVEL NON-INVASIVE BIOMARKERS}

\begin{abstract}
1,23,4 HM Gunn*, 3,4VS Forsyth, 1f Hällqvist, ${ }^{2} \mathrm{R}$ Viner, ${ }^{1} \mathrm{~K}$ Mills, 3,4KS Steinbeck. ${ }^{1}$ Translational Mass Spectrometry Research Group, UCL Great Ormond Street Institute of Child Health, London, UK; ${ }^{2}$ Population, Policy and Practice Programme, UCL Great Ormond Street Institute of Child Health, London, UK; ${ }^{3}$ Academic Department of Adolescent Medicine, Sydney Children's Hospital Network, Sydney, Australia; ${ }^{4}$ Discipline of Child and Adolescent Health, The University of Sydney, Sydney, Australia
\end{abstract}

\subsection{6/bmjpo-2019-RCPCH-SAHM.6}

Background Polycystic ovarian syndrome (PCOS) is common, affecting up to one-fifth of females and is associated with significant comorbidity. Despite this, it is poorly understood, and diagnosis and management remain challenging in adolescents. Proteomics enables the better understanding of disease mechanisms and facilitates the identification of novel biomarkers. Objectives To describe the clinical phenotype of PCOS in adolescents and undertake discovery proteomic urine profiling using ultra-performance liquid chromatography-mass spectrometry (UPLC-MS/MS) to identify novel non-invasive biomarkers of PCOS.

Method This prospective longitudinal study recruited adolescent females meeting NIH diagnostic criteria for PCOS. The following were measured at baseline and annual follow-up: hormonal and metabolic markers including an oral glucose tolerance test, psychological, pubertal and anthropometric parameters, and pelvic ultrasounds. We have undertaken UPLC-MS/MS and developed new methods for discovery proteomic profiling of urine in an attempt to identify new disease mechanisms, drug targets and potential biomarkers.

Results We recruited 40 participants (median age 15.0 years, range 12.5-18.3), with two-thirds completing annual followup. Clinical signs at presentation included acne (89\%), hirsutism (78\%) and acanthosis nigricans (49\%). Two-thirds of participants had depressive or anxiety symptoms yet only one-third were known to mental health services. Metabolic dysfunction was common at baseline; overweight/obesity $(86 \%)$, elevated body fat (88\%) and dyslipidaemia (35\%). These parameters persisted at follow-up. Insulin resistance was almost universal at baseline and follow-up (91\%). Impaired glucose metabolism was common but improved from baseline $(29 \%)$ to follow-up $(10 \% ; p=0.11)$. Over two-thirds had elevated anti-Müllerian hormone, three-quarters had an elevated free androgen index. Raised inflammatory markers (CRP/ESR) were present in $40 \%$ participants. Only three participants had definitive ultrasonographic evidence of PCOS. Interventions included lifestyle advice $(27 \%)$, combined oral contraceptive pill (COCP) \pm antiandrogen (16\%), metformin (30\%) or metformin + COCP \pm anti-androgen (27\%).

Conclusion and future directions Adolescents withPCOS are at high risk of metabolic dysfunction, inflammation and mental health disorders. Therefore, early diagnosis and intervention are imperative. However, current diagnostic and surveillance methods are suboptimal. We have used urinary proteomics to study metabolic pathways affected in PCOS and aim to identify novel non-invasive biomarkers. Subsequently, we will create a clinically translatable assay to aid diagnosis and stratify management of this common adolescent condition.

\section{YOUTH GAMBLING AND MENTAL HEALTH- A POPULATION STUDY}

${ }^{1} \mathrm{~A}$ Emond*, ${ }^{2} \mathrm{M}$ Griffiths, 'L Hollen. 'Centre for Academic Child Health, Bristol Medical School, Bristol, UK; ${ }^{2}$ International Gaming Research Unit, Nottingham Trent University, Nottingham, UK

\subsection{6/bmjpo-2019-RCPCH-SAHM.7}

Aims To investigate gambling behaviour in youth aged 17-24 and explore the associations with mental health and wellbeing.

Methods A large contemporary UK cohort study, the Avon Longitudinal Study of Parents and Children (ALSPAC), was used to collect the data. Young adult participants completed computer-administered gambling surveys in research clinics, on paper and online. Depression, anxiety and wellbeing scores, and drug and alcohol usage, were collected by selfcompletion questionnaires. The sample sizes were 3566 at age 17 years, 3940 at 20 years, and 3841 at 24 years. Multiple imputation techniques were utilised to adjust for missing data, and multivariable models created using the imputed data set.

Results Participation in gambling in the last year was reported by $54 \%$ of 17 -year-olds, rising to $68 \%$ at 20 years, and $66 \%$ at 24 years, with little overall variance. Regular (weekly) gambling showed a strong gender effect, increasing from $13 \%$ at 17 to $17 \%$ at 24 years. The commonest forms of gambling were playing scratchcards, playing the lottery, and private betting with friends. The only activity which increased markedly between 17 and 24 years was gambling on activities via the internet, especially in males.

Problem gambling was measured at each age using the Problem Gambling Severity Index (PGSI), and responses categorised into 'no problem' 'low risk gambling' (16-21\%) and 'moderate risk/problem gambling' (6-7\%). At risk gamblers had shown higher hyperactivity scores and conduct problems on the SDQ at 16 years. Between 17 and 24 
years, any at risk gambling was associated with higher depression and anxiety scores, and wellbeing scores in the lowest quartile. The adjusted odds ratios (95\% CI) were highest in the moderate/problem group at 20yrs: depression 2.29 (1.28, 4.12), low wellbeing 1.61 (1.01, 2.57), involvement in crime $2.47(1.54,3.97)$, problematic use of alcohol $2.64(1.13,6.17)$, and drug use 1.79 (1.16, 2.75). Problem gamblers were more likely to have parents who gambled.

Conclusion Although many young people gamble without any harm, a significant minority (mainly males) show problem gambling behaviours which are associated with poor mental health, low wellbeing, and potentially harmful use of drugs and alcohol.

\section{A BIOPSYCHOSOCIAL MODEL OF CARE FOR CHILDREN AND YOUNG PEOPLE (CYP) WITH PERSISTENT, UNEXPLAINED, PHYSICAL SYMPTOMS (PUPS)}

1J Pales*, ${ }^{1} \mathrm{~K}$ Street, ${ }^{1} \mathrm{R}$ Howells, ${ }^{2} \mathrm{~A}$ Lee, ${ }^{2} \mathrm{~J}$ Burrows, ${ }^{3} \mathrm{~A}$ Boyd, ${ }^{3} \mathrm{~T}$ Bloomfield, ${ }^{4} \mathrm{~V}$ Palfrey. ${ }^{1}$ Paediatrics, Royal Devon and Exeter Foundation NHS Trust, Exeter, UK; ${ }^{2}$ Child and Adolescent Mental Health Service, Virgin Care Ltd, Exeter, UK; ${ }^{3}$ Psychology, Virgin Care Ltd, Exeter, UK; ${ }^{4}$ School Inclusion Team, Devon County Council, Exeter, UK

\subsection{6/bmjpo-2019-RCPCH-SAHM.8}

Aims PUPS are common, reported by $10-25 \%$ of CYP. ${ }^{1}$ Symptoms can lead to poor function, overuse of medical resource and reduced school attendance. Co-morbid mental health problems often go unrecognised. Longer term outcomes include adult chronic physical/mental ill-health, reduced employment, high health/welfare costs. ${ }^{2} 3$ We established a pilot multi professional assessment/support service to meet the needs of these CYP.

Methods Weekly multiprofessional meetings including paediatrician, psychiatrist, CAMHS worker, psychologist and education wellbeing advisor (EWA) to discuss cases referred by health professionals. Patient/parent consent given. Outcomes included holistic paediatric assessment, joint appointments (paediatrician and CAMHS worker/psychologist), advice and guidance (A\&G) to referrer such as signposting/facilitated referral to community services. Some were offered short-term therapeutic intervention with CAMHS worker/psychologist and/or psychiatric assessment. In all cases clear communication with school was facilitated by EWA who supported school attendance; assisting re-integration and improved attendance/ wellbeing at school.

Results Over 18 months we discussed 180 patients: 74 male, 104 female, 2 transgender. Average age 14 years. Common PUPS were musculoskeletal pain, fatigue, headaches, abdominal pain and unexplained episodes. All had reduced school attendance. 111 cases referred by Paediatricians/Allied Health professionals, 56 new GP referrals, 13 presented acutely. 106 were offered paediatric appointments. $>50 \%$ were discharged with recommendations/advice to primary care/education. $25 \mathrm{had}$ joint appointments. 38 were seen by psychiatrist/CAMHS worker/psychologist for assessment/therapeutic intervention. Remainder received A\&G and EWA support. Cost analysis demonstrated average savings of $£ 2600 /$ patient in secondary care. School attendance improved for the majority with reintegration plans and reduction in use of out of school provision with associated cost savings. Referrals to tertiary services for chronic pain/fatigue were reduced and joint working with these services was developed. Linked case examples show significant improvement.

Conclusion Multiprofessional assessment using a biopsychosocial approach to CYP with PUPS leads to better recognition of underlying mental illness, improved short-term functional outcomes, reduced medical costs and improved school attendance. The challenge is securing longer term funding

\section{REFERENCES}

1. Hinton D, Kirk S. Families' and Healthcare Professionals' perceptions of healthcare services for children and young people with medically unexplained symptoms: a narrative review of the literature. Health \& social care in the community 2016;24 (1):12-26.

2. Campo JV. Annual Research Review: Functional somatic symptoms and associated anxiety and depression - developmental psychopathology in pediatric practice. Journal of Child Psychology and Psychiatry 2012;53:575-592.

3. Bermingham SL, Cohen A, Hague J, Parsonage P. The cost of somatisation among the working-age population in England for the year 2008-2009. Mental Health in Family Medicine 2010;7:71-84.

\section{P1 $\quad$ SOCIO-CULTURAL PERSPECTIVES ON CONDOM USAGE INTENTIONS AND BEHAVIOURS AMONGST AFRICAN ADOLESCENT MIGRANTS IN SOUTH AFRICA}

${ }^{1}$ RR Titus*, ${ }^{2}$ LL John-Langba. 'Social Development, University of Cape Town, Cape Town, South Africa; ${ }^{2}$ School of Applied Human Science, University of Kwazulu-Natal, KwazuluNatal, South Africa

\subsection{6/bmjpo-2019-RCPCH-SAHM.9}

Background Adolescent health has become a critical issue in the HIV intervention discourse as this particular cohort has an increased vulnerability to HIV infection, however migrant youth has an additional vulnerability due to their migrant status. African adolescents and migrant status are key population cohorts within South Africa identified as increased risk of HIV infection, sexual transmitted infection and reproductive health problems. Although increased focus into migration patterns in the South African context, not much research focused on the adolescent health of migrants. The impact of socio-cultural perspectives on condom use intentions and behaviour on migrant youth, which translate to the effect that social and cultural dynamics have on decision making processors of young people with regard to their sexual health choices and risk patterns of HIV and reproductive health.

Objective We examined migrant adolescent experiences of condom use in South Africa and identified socio-cultural factors influencing the condom use intentions and behaviours of migrant youth.

Method This qualitative study employed purposive and snowballing sampling techniques to explore with migrant youth regarding their intentions and behaviour towards condom use within their social cultural contexts. The sample included 20 African descent adolescents from Sub-Saharan African, notably from Zimbabwe, Nigeria, Congo, Cameroon, Kenya and Malawi. The mean age was 23 years with a range between 20 years to 25 years.

Results Findings indicated that young migrants have a good understanding of the functions of condoms and the dual protection against pregnancy and sexually transmitted infections. However, the use of condoms, even freely available, is a contested issue as socio-cultural practices and influences have an impact on the intentions and behaviour towards condom use among young migrant adolescents. 